\title{
The Development of Chinese Language and Literature Major Under the Background of Big Data
}

\author{
Xiaofang Yuan ${ }^{1, a}$ \\ ${ }^{1}$ Yichun Early Childhood Teachers College, Jiangxi, Yichun, 336000 \\ a406659385@qq.com

\begin{abstract}
At this stage, under the background of the rapid development of network technology, the ways and means of information transmission have become more abundant. Our society should conform to the development trend of the times and enter the era of big data. In the era of big data, all people's social activities can not leave the network, and information dissemination and convergence are becoming more and more rapid. In this context, the professional development of Chinese language and literature has encountered more opportunities and challenges. This paper aims at how to avoid the impact of the new environment, conform to the trend of network development, optimize and expand the prospects of Chinese language and literature, and launch a series of reasonable analysis, as a reference
\end{abstract}

Keywords: big data era, Major in Chinese language and literature, teaching

\section{浅谈大数据背景下汉语言文学专业的发展}

\author{
袁晓芳 ${ }^{1, a}$
}

\author{
${ }^{1}$ 宜春幼儿师范高等专科学校 江西 宜春 336000 \\ a406659385@qq.com
}

\section{摘要:}

现阶段, 在网络技术快速发展的背景下, 信息传递的方式和途径变得更加丰富, 我国社会应该顺应时代发展 潮流步入大数据的时代。在大数据的时代背景下, 人们所有的社会活动基本都不能离开网络, 信息传播和交 汇逐渐变得更加迅速。在如此背景之下, 汉语言文学专业发展过程中, 遇到了更多的机遇和挑战, 文章针对 该怎样应对新环境下出现的冲击, 顺应网络发展趋势, 对汉语言文学的前景进行优化和拓展, 展开了一系列 的合理分析, 以此作为参考。

关键词: 大数据时代；汉语言文学专业；教学

\section{1. 大数据的内涵特点与汉语言文学专业概述}

\section{1. 大数据概述}

大数据 (big data), 是一种新型的数据处理技 术形式，对于平时时间中没有办法借助正常手段进行 处理以及捕捉的数据集合, 以强大的算力为基础, 针 对这些数据展开综合分析以及处理的一种技术。简而 言之, 数据分析是大数据技术的核心功能及作用, 根 据分析得出的结果, 对有关决策进行辅助, 或者是实 现它和智能决策的相互融合, 把数据分析作为实现自
动决策的依托。针对目前大数据的发展状况, 它已经 被应用在很多领域, 同时也在很多地方得到了实现, 例如智慧交通、电子商务、电力供应、人口流动以及 物流运输等层面, 都逐渐形成了有关的大数据体系。 把运用大数据技术作为依托, 让从前没有得到重视并 且不好被利用的数据, 渐渐显现出了重要价值。

对大数据的发展历程进行观察并研究, 它局部中 许多层面的重要特点, 这些特点对所有行业的发展都 产生了比较重要的借鉴作用。

首先, 多样性。大数据技术包含的数据多种多样, 不是从单个方面开展数据的收集和分析工作的, 而是 
尽可能多的收集更加全面的数据信息, 所以在开展数 据处理的过程中, 会和很多各种各样的数据产生关联, 进一步表现出多样性的特征。其次, 信息化。实际上, 大数据技术也是信息技术的一种。大数据技术的不断 发展和快速进步，推动了社会信息化的发展进度。例 如, 大数据在建设智慧城市的工作中发挥了极大的作 用，在其中表现出的信息化特征，是人们应该关注的 重点。再次, 真实性。数据的真实程度也是大数据技 术的一大特征。在使用大数据技术的过程中要得到具 体的成就就要确保数据的真实程度, 数据来源是否可 靠, 数据是否真实合理是不能忽视的。另外, 利用大 数据技术开展数据分析工作的过程中, 会判断数据的 真实性, 在对数据进行分析的过程中, 排除掉一些不 真实的数据, 从而增强数据的可信程度。

\section{2. 汉语言文学专业概述}

\section{2. 1. 汉语言文学专业的定义}

在普通高等学校中, 汉语言文学属于本科专业, 是属于中国语言文学类的一种专业, 它的基本修业年 限是四年, 在四年的学习之后, 学生会被授予文学学 士的学位。这个专业致力于培养文学素养以及语言文 学基础较高的学生, 应该很好地学习中国语言文字的 相关基础知识 应该培养成较高的高等文字解决能力、 文献书籍阅读能力等高水平人才。

\subsection{2. 关于汉语言文学专业的发展方向}

新闻编辑、师范、秘书学、书法学自己人文旅游 这五个方面是现阶段我国汉语言文学专业的发展方 向, 依靠不同的培养计划开展不同特征的教学实 践, 让学生在适合自己的社会岗位中提现自己的价 值, 例如新闻、教育以及政治等相关工作岗位, 将 文学作为工作的重点, 汉语语言文学给社会发展提 供更多的高质量高素质人才。

\section{2. 大数据时代给汉语言文学专业带来的挑战}

\section{1. 网络语言与新名词的冲击}

随着大数据的迅速发展, 网络语言也迅速在大众 生活中产生, 网络语言具有其自身的特点, 依靠简洁 生动的语言出现在各种媒体平台上, 同时在生活交流 中出现的频率也很高, 越来越多的人们开始关注网络 语言, 使用网络语言, 网络语言以及一些新词在人们 的日常生活中占据不小的地位。

这样的全新产生的语言文字带来的影响有好有 坏, 好处是流行语言和新型词汇是建立在汉语上的, 可以丰富汉语的内涵, 让汉语与时俱进, 在汉语中不 断注入新鲜的生命力, 这些流行语言和新型词汇可以 准确的表达人们对现在事实的一些想法以及考虑; 但 是也有消极的一面, 由于没有进过专业学者和专家的
商讨, 这些词汇有很多错误和表达不准确的地方, 错 误的语音和词汇会导致语言系统紊乱，让破坏了文学 艺术的准确性和专业性受到破坏，导致语言过于娱乐 化, 缺乏实际意义。

\section{2. 汉语言文学专业教学知识与大数据社会 脱节}

现在大数据更新换代速度很快, 在当前的背景下, 所有行业都要紧跟时代的步伐, 否则一旦落后, 就很 容易被市场淘汰, 过去的学习汉语的学生的竞争力下 降, 应该要创建全新的课堂模式。现在我们国家高等 院校的汉语言课程还是过于老套, 教授的内容都是过 时的, 落后陈旧的, 没有加入新鲜血液, 陈旧的思维 模式会严重阻碍学生深入了解现在的社会, 学生难以 了解到现实社会, 没有让学生增加自身的学问和知识。 这导致汉语言专业的学生不适应现在的就业环境, 不 容易找到心仪的工作, 也无法为社会, 为国家提供更 好的价值, 这些问题的存在让汉语言专业更加没落, 显现出很多缺陷, 只有解决了这些阻碍汉语言才能够 繁荣起来。

\section{3. 大数据时代给汉语言文学专业带来的机遇}

\section{1. 大数据发展带来的专业新出路}

互联网已经遍布世界各个角落，让全世界信息 交流速度大大提升, 人们可以更加方便快捷的阅读 各种文字, 新媒体行业发展的越来越好, 这说明语 言类专业学生有了新的就业方向。写文章发表在网 络平台, 写策划方案, 促进销售都可以让汉语言人 才进入新的行业, 高等院校的学生们的知识水平比 互联网作者要高很多, 如果高等院校学生能进入这 些行业, 那么汉语言文学专业的就业前景就更加乐 观。

\section{2. 大数据的发展是新汉语言文学专业教育 模式发展的动力}

步入二十一世纪以后，随着互联网技术的不断发 展, 我国逐渐步入了大数据时代, 互联网技术和大数 据信息在社会发展的过程中发挥着至关重要的作用。 高校在开展汉语言文学教育时需要充分发挥出互联 网技术和大数据的价值, 教师也可以通过大数据平台 来与其他高校的教师进行交流沟通，以此来借鉴对方 的长处, 教师应当不断调整自身的教学方式, 为学生 提供高质量的课堂教学。除此之外, 教师在开展汉语 言专业教学时还需要利用大数据来创新自身的教学 方式, 比如: 在疫情期间, 教师可以通过互联网技术 来开展线上教学, 将所学的内容制作成视频传到网络 平台上, 使学生可以通过线上教学来进行自主学习, 以此来提高学生的自主学习能力和独立思考能力, 这 种教学方式可以促进学生进行全面发展。 


\section{3. 大数据的发展带动了汉语言专业的进步}

科学技术的进步让汉语言文学的发展和教学产 生了巨大的改变, 信息的飞速传输稳固了汉语言文学 专业和大数据时代的关联, 从而形成了互补的干系。 文字中蕴含着巨大的能量, 其中包含着一个民族的灵 魂, 汉语言文学对社会的进步起到了至关重要的作用, 并且还可以在大数据的发展中汲取精华推动自身进 步。互联网的产生也使我们了解到了其他的民族语言 的魅力, 以大数据为基础创建国际性语言平台, 可以 推动汉语言文学在平台中了解其他语言的特色, 从而 丰富自身的语言文化。大数据还可以让如今的汉语言 文学更加充分的了解中国传统的古文化, 经过互联网 中的相关书籍去了解中华民族上下五千年的语言文 化。互联网将过去和未来结合起来, 相连中国和世界, 从而促进了汉语言文学的进步, 扩大了汉语言文学专 业的进步和创新的道路。

\section{4. 大数据时代影响汉语言文学专业教学的措 施研究}

在大数据时代发展的背景下, 如今的汉语言文学 专业逐渐显露出了部分问题, 课程老师应该要对这些 问题存在的原因有自身的了解, 其次掌握大数据时代 的发展特征, 对于教学模式进行创新升级从而强化教 师的汉语言文学专业的教学质量水平。

\section{1. 运用多样化方式展开教学}

汉语言文学专业的教学模式要多样化和多元化, 开拓不同形式的课堂教学方式。让课堂教学更加具有 生动性和趣味性, 增加学生在课堂上的参与程度, 让 学生真正融入到课堂学习中。可以从以下几点展开: 第一, 开展问题清单导学。问题清单导学是一种以教 学内容为基础的问题清单。教师在课堂上根据清单上 所列的问题, 来逐步引导学学生。例如, 在讲授中国 当代文学时, 像《骆驼祥子》, 可以依据课文内容来 构造有关问题, 在这些问题的引导下让学生有目的性 得去学习, 带着问题去把握文章, 理解文章整体内容 和中心思想。第二, 开展趣味活动。在教师讲授文学 作品时, 课堂形式不能被教师的讲解所占用, 还应该 穿插一些趣味活动。使得学生在这些趣味活动中, 深 刻理解文学中的人物形象和故事情节。例如在讲授 《阿 $\mathrm{Q}$ 正传》时, 可以依据阿 $\mathrm{Q}$ 的精神胜利法, 实施 情景再现活动。让学生在体会阿 $\mathrm{Q}$ 精神的基础上表演 阿 $Q$, 深刻理解阿 $Q$ 的内心想法, 形成对精神胜利法 更深理解。第三, 开展小组合作学习。教师可以在课 堂上将学生分为几个小组, 共同对课文内容进行沟通 和研究。

\section{2. 运用信息化方式进行教学}

在开展不同形式的教学活动时, 信息技术的运用 还要得到重视, 借助一些信息化手段来提升教学质量
和效果。首先, 借助信息化微课辅助教学。微课时近 几年来热度逐步增加的教学方式, 承载了教学的视频 和资料, 让视频联系上课所学内容。可以将制定学习 微课的方法应用到汉语言文学专业的教学实践中。在 课堂上借助多媒体来播放微课, 让学生观看微课, 进 行自主学习。还可以建立起完善、规范的网络教学平 台。就汉语言文学专业的教学活动来说, 还可以以信 息技术为基础, 创建网络教学平台, 把教学活动和实 践活动相结合。例如: 可以以微信小程序为基础, 创 建汉语言文选专业的学习程序, 在程序中可以建立各 种学习服务功能, 使学生可以经过手机微信进行汉语 言文学学习。

\section{3. 通过利用数据发掘技术来提高学生评价 结果的真实性}

教师和学校在开展汉语言教学时还需要认识到 学生评价的重要性, 需要充分发挥出学生评价的价值。 为了有效提高学生评价学生结果的真实性, 教师和学 校可以采用数据发掘技术, 比如: 教师在考核评价学 生的平时成绩时可以利用数据发掘技术, 对学生的课 堂出勤率、课堂小测试、期末考试等进行详细的考核 总结, 然后将这些成绩做成与考核相关的数据信息, 建立起完善的数据库。与此同时, 教师还可以利用数 据挖掘技术来建立起科学的评价标准, 然后根据这些 标准来进行考核评价工作, 以此来提高学生评价工作 的准确性和真实性, 使教师可以更加深入地了解学生 的学习情况。

\section{5. 结论:}

随着大数据时代的飞速发展, 针对大数据时代的 发展所引起的惊涛骇浪, 汉语言文学专业必须要立足 于实践, 心系未来，对于时代的发展要 “取其精华、 弃其糟粕”。保持积极的发展心态, 面对挑战和机遇 要勇往直前, 以信息化数据化的发展浪潮为踏板坚定 自身的脚步。

\section{REFERENCES}

[1] Yang Xiaoyu. Optimization strategies of Chinese language and Literature Teaching in the new media environment — a review of teaching research and Reform Exploration of Chinese language and literature [J]. News front, 2017, 0 (12x): 147-147

[2] Chen Hongyan. A new exploration of "modern Chinese" teaching for " $2+2$ " TCFL major in Sino Korean cooperation [J]. Journal of Anqing Normal University (SOCIAL SCIENCE EDITION )， 2011，30 (8): $114-118$

[3] Xia Congcong. Informatization development of modern Chinese language and literature and talent cultivation of students [J]. Journal of Taiyuan City Polytechnic, 2014, 0 (2): 135-136 
[4] Ma Yupeng. One belt, one road initiative, the internationalization of Guangxi's universities and Colleges: a case study of Guangxi Foreign Languages College, [J]. era report: Academic Edition, 2019, 0 (5): $230-231$.

[5] Xiong shaogao. Using information technology to improve non intelligence factors of adult students Taking Chinese language and literature major as an example $[\mathrm{J}]$. Contemporary education theory and practice, 2012, 4 (4): 59-61 